among women and it is even higher in HIV-infected women. Our goal was to determine the prevalence rate of High-risk (HR) HPV and associated factors among HIV-infected women attending referral care centres for HIV/AIDS in different regions of Brazil.

Methods Cross-sectional study conducted among HIV-infected women attended at referral care centres for HIV/AIDS in nine states of Brazil. Women from 18 to 49 years that accept to participate and were not pregnant at the time of the approach were recruited for the study. The HPV screening was realised using qPCR in closed system, In vitro Diagnostic, COBASHPV Roche. The cytology results were available by the Bethesda System.

Results A total of 802 (89.1\%) women participated. Median age was 39 (Inter quartile range (QR34-46)) years and median education was 9 (IQR6-11) years. The general prevalence of HR-HPV was $28.4 \%$ (228/802). The prevalence rate of HPV16 was $8.1 \%(65 / 802)$, HPV 18 was $3.7 \%(30 / 802)$ and other types of HR-HPV were $23.6 \%$ (189/802). The factors associated with HR-HPV in the multivariate logistic regression analysis were: age ranging from 18 to 34 years $[\mathrm{OR}=1.43(95 \%$ CI:1.18-1.75)], drug abuse $[\mathrm{OR}=1.61 \quad$ (95\%CI:1.10-2.42)] and abnormal cervical cytology $[\mathrm{OR}=1.56$ (95\%CI:1.341.81)].

Conclusion Results showed high prevalence of high-risk HPV infection in women living with HIV in Brazil. The infection was significantly associated with age less than 35 years old, illicit drug use and abnormal cervical cytology. HR-HPV test in HIV-seropositive women is a useful procedure to implement cervical cancer screening.

Support: Technical cooperation agreement, Brazilian Department of STI, AIDS and viral hepatitis, Ministry of Health and United Nations office for drugs and crime. Project BRA/K57, process \#01/2013

\section{P3.14 PREVALENCE OF CHLAMYDIA TRACHOMATIS AND NEISSERIA GONORRHOEA AND ASSOCIATED FACTORS AMONG WOMEN LIVING WITH HUMAN IMMUNODEFICIENCY VIRUS IN BRAZIL: A MULTICENTER STUDY}

\begin{abstract}
${ }^{1}$ Angelica E Miranda, ${ }^{2}$ Mariangela F Silveira, ${ }^{3}$ Ana Gabriela Travassos, ${ }^{4}$ Teresinha Tenorio, ${ }^{5}$ Isabel Cristina Chulvis Do Val, ${ }^{6}$ Leonor Lannoy, ${ }^{7}$ Hortensio S Mattos-Junior, ${ }^{8}$ Newton Sergio de Carvalho. 'Universidade Federal do Espirito Santo - Vitoria - ES, Brazil; ${ }^{2}$ Universidade Federal de Pelotas, Pelotas - RS, Brazil, ${ }^{3}$ Universidade Estadual da Bahia, Salvador - BA, Brazil; ${ }^{4}$ Universidade Federal de Pernambuco, Recife - PE, Brazil; ${ }^{5}$ Universidade Federal Fluminense, Niterói - RJ, Brazil, ${ }^{6}$ Unidade de Saúde Mista da Asa Sul, Brasilia - DF, Brazil; ' Laboratório São Marcos, Vila Velha - ES, Brazil; ${ }^{8}$ Universidade Federal do Paraná, Curitiba - PR, Brazil
\end{abstract}

\subsection{6/sextrans-2017-053264.251}

Introduction Chlamydia trachomatis (CT) and Neisseria gonorrhoeae (GC) cause infections in the female genital tract, increasing susceptibility to and infectiousness of HIV. Our objectives were to determine prevalence and associated factors of CT and GC among HIV-infected women in Brazil.

Methods Cross-sectional study, including HIV-infected women attending nine referral centres in nine states of Brazil, aged 18 to 49 years, not pregnant. An interview was conducted including sociodemographic, epidemiological and clinical characteristics. After the interview, gynaecological examination was conducted to collect cervical cytology and vaginal secretion to
Chlamydia trachomatis, Neisseria gonorrhoeae and HPV tests through molecular biology.

Results A total of $802(89.1 \%)$ women participated. The prevalence of CT was 17 cases (2.1\%) and GC was 7 cases $(0.9 \%)$. The prevalence of a positive test for both CT and/or GC was $2.7 \%$. The factors associated with positive CT/GC test in the multivariate logistic regression analysis were abnormal papanicolau smear [OR 4.1 (95\% CI:1.54-11.09)] and the presence of abnormal cervical discharge $[\mathrm{OR}=2.6 \quad(95 \%$ CI:1.02-6.71)]. Among the 377 women that reported previous STI: $245(65.0 \%)$ reported using condom more frequently after being diagnosed. Regarding how they discovered the STI, $62(16.4 \%)$ the partner told he was infected by an STI; 157 $(41.6 \%)$ had STI symptoms and looked for care and 158 $(41.9 \%)$ discovered it in a routine consultation for another reason.

Conclusions The control of STI represents a unique opportunity to improve reproductive health of women living with HIV. This diagnostic can change their behaviour and reduce the sexual transmission of HIV and bacterial STI. Controlling STI and identifying factors associated with such diseases continues to be an important element in the design of interventions targeting STI and as a result, HIV prevention in Brazil.

Support: Technical cooperation agreement, Brazilian Department of STI, AIDS and viral hepatitis, Ministry of Health and United Nations office for drugs and crime. Project BRA/K57, process \#01/2013

\section{P3.15 UNPROTECTED SEX WITH NON-COMMERCIAL PARTNERS AS THE MAIN RISK FACTOR TO GET STI FOR FEMALE SEX WORKERS IN ARMENIA}

A Asmaryan, S Grigoryan, E Hovhannisyan. National Centre for AIDS Prevention, Republic of Armenia

\subsection{6/sextrans-2017-053264.252}

Introduction HIV Biological and Behavioural Surveillance surveys (BBSS) were conducted among female sex workers (FSW) in Armenia in 2010, 2012, and 2014. These surveys used respondent driven sampling (RDS), an effective method for recruiting hidden populations.

Methods In 2016 the BBSS using RDS among FSW were conducted in Yerevan the capital of Armenia. The sample size was 300. Prevalence of HIV, syphilis, Trichomoniasis, Neisseria gonorrhoeae (NG) and Chlamydia trachomatis (CT) were measured and knowledge and sexual risk behaviours were assessed.

Results HIV prevalence was low at $0.1 \%$ among FSW in Yerevan. NG prevalence was $4 \%, 29 \%$ of FSW were positive for Trichomoniasis. The prevalence of CT was 12\%, syphilis prevalence was 4\%. 34\% of FSW in Yerevan reported having had genital ulcers or sores in the past 12 months.

More than 95\% of FSW in all survey locations reported using condoms the last time they had sexual intercourse with a client. $89 \%$ of FSW in Yerevan reported sex with non-commercial partners in the past 12 months and 38\% reported not using condoms during their last sex with them.

Conclusion There was a significant increase from $90.2 \%$ in 2012 to $99.6 \%$ in 2016 in the use of condoms with the most recent client among FSW. But there was no overall significant change in STI prevalence among FSW between 2012 and 2016. Many FSW reported having sex with non-commercial 